How to cite this article:

Shanmugasundaram, K., Mohmed, A. S. A., \& Ruhaiyem, N. I. R. (2019). Hybrid improved bacterial swarm optimization algorithm for hand-based multimodal biometric authentication system. Journal of Information and Communication Technology, 18(2), 123-141.

\title{
HYBRID IMPROVED BACTERIAL SWARM OPTIMIZATION ALGORITHM IN HAND-BASED MULTIMODAL BIOMETRIC AUTHENTICATION SYSTEM
}

\author{
Karthikeyan Shanmugasundaram, Ahmad Sufril Azlan Mohmed \\ \& Nur Intan Raihana Ruhaiyem \\ School of Computer Sciences, Universiti Sains Malaysia, Malaysia
}

karthik_mcamtech@rediffmail.com;sufril@usm.my;intanraihana@usm.my

\begin{abstract}
This paper proposes a Hybrid Improved Bacterial Swarm (HIBS) optimization algorithm for the minimization of Equal Error Rate (EER) as a performance measure in a hand-based multimodal biometric authentication system. The hybridization of the algorithm was conducted by incorporating Bacterial Foraging Optimization (BFO) and Particle Swarm Optimization (PSO) algorithm to mitigate weaknesses in slow and premature convergence. In the proposed HIBS algorithm, the slow convergence of $\mathrm{BFO}$ algorithm was mitigated by using the random walk procedure of Firefly algorithm as an adaptive varying step size instead of using fixed step size. Concurrently, the local optima trap (i.e. premature convergence) of PSO algorithm was averted by using mutation operator. The HIBS algorithm was tested using benchmark functions and compared against classical $\mathrm{BFO}$, PSO and other hybrid algorithms like Genetic AlgorithmBacterial Foraging Optimization (GA-BFO), Genetic AlgorithmParticle Swarm Optimization (GA-PSO) and other BFO-PSO algorithms to prove its exploration and exploitation ability. It was observed from the experimental results that the EER values, after the influence of the proposed HIBS algorithm, dropped to $0.0070 \%$ and $0.0049 \%$ from $1.56 \%$ and $0.86 \%$ for the right and left hand images of the Bosphorus database, respectively. The
\end{abstract}


results indicated the ability of the proposed HIBS in optimization problem where it optimized relevant weights in an authentication system.

Keywords: Bacterial foraging, particle swarm optimization, firefly algorithm, biometric authentication system.

\section{INTRODUCTION}

The hand-based multibiometric system is a promising approach in multibiometric authentication due to its ease of use, low cost and reliability (Ross, Nandakumar, \& Jain, 2006). The hand-based multibiometric system is used in various real time systems like immigration, border security, law enforcement and forensics, user entry access system, financial transaction and more. Moreover, the use of evolutionary based fusion in multibiometric system is a promising state-of-the-art approach and has proven its ability in improving performance accuracy compared to deterministic and probabilisticbased fusions. Further, the issue of low accuracy in hand-based multibiometric system has also been addressed (Jain, Nandakumar, \& Ross, 2016). Swarm Intelligence (SI) based hybrid meta-heuristic algorithm (Kennedy, Kennedy, Eberhart, \& Shi, 2001) has been used to resolve the issue of low accuracy by optimizing weights associated with hand-based modalities.

In this paper, the proposed algorithm is used to mitigate the weaknesses of BFO (Passino, 2002) and PSO (Eberhart \& Kennedy, 1995) algorithms that include slow and premature convergence (Shanmugasundaram, Mohamed, \& Ruhaiyem, 2017a). At the score fusion (Ross et al., 2006), the hand-based multimodal biometric traits like fingerprint, palm print and finger inner knuckle print are fused along with optimal weights induced by the hybrid algorithm which minimizes error rates.

\section{RELATED WORK}

Score level fusion using hybrid GA-PSO optimization techniques have been used to optimize weights associated with fused modalities to get optimum EER values (Cherifi Dalila, Hafnaoui Imane, \& Nait-Ali Amine, 2015). However, the PSO algorithm suffers from premature convergence hence it has affected performance accuracy to a great extent. On the other hand, genetic and evolutionary computations for multimodal biometrics using score level fusion have produced better accuracy (Alford et al., 2011). To select optimal parameters, a hybrid PSO algorithm is employed in decision level fusion 
carrying of two modalities: palm print and hand geometry, respectively. In the hybrid PSO algorithm, continuous PSO is used for calculating updates of the position and velocity of a particle and binary PSO is utilized for attaining a fusion rule (Gabi, Ismail, Zainal, Zakaria \& Al-Khasawneh, 2018; Hanmandlu, Kumar, Madasu, \& Yarlagadda, 2008).

Biswas, Das and Abraham (2007) proposed a hybrid BFO-PSO algorithm in order to increase convergence speed and accuracy of the BFO algorithm. In the study, PSO algorithm was used as a mutation operator to attain the best value. This algorithm had shown efficiency in solving multimodal optimization problems (Shehab, Khader \& Laouchedi, 2018).

The Bacterial Foraging Optimization-parameter free Particle Swarm Optimization (BFO-pfPSO) algorithm was proposed by Bakwad et al. (2009). In the algorithm, all bacteria positions and directions were updated after all fitness evaluations had been completed, instead of after each fitness evaluation. The BFO upgraded its current position by parameter free PSO (pfPSO) to accelerate global performance of BFO. Hence, updates on velocity, inertia weights, and acceleration constants were not required.

Yan, Zhu, Chen, and Zhang (2012) proposed an Improved Bacterial Foraging Optimization (IBFO) algorithm where social co-operation was introduced for guiding bacteria tumbling towards better directions. In addition to that, an adaptive step size was adjusted in descending order. Later, ACBSFO_DES algorithm (Jarraya, Bouaziz, Alimi, \& Abraham, 2013) was proposed where the BFO algorithm was hybridized with the PSO algorithm for velocity updates. The crossover DE was used for position and adaptation at the step size of chemotaxis stage in the $\mathrm{BFO}$ algorithm.

In 2013, Alostaz and Alhanjou proposed the ABFO_PSO algorithm. The proposed study used the BFO algorithm to adjust step size in order to calculate the magnitude of the velocity of the particle in PSO. The hybrid algorithm of the BFO-PSO used feature selection algorithm to detect bundle branch block in which the size of the database used was gradually reduced (Kora \& Kalva, 2015). However, classifier training time might also be increased. Daas, Chikhi, and Batouche (2015) proposed the FABFO algorithm which eliminated dispersal and reproduction steps found in the BFO algorithm. Such an approach increased convergence speed and reduced time complexity.

\section{PROPOSED METHOD}

The methodology of the study consisted of two phases: i) implementation of Hybrid BFO-PSO (HIBS) algorithm (Shanmugasundaram et al., 2017a) 
and ii) deployment of Hybrid BFO-PSO (HIBS) algorithm in a hand-based multibiometric authentication system. The role of the hybrid algorithm was to select optimal weights at the score fusion which involved error minimization (EER) as the performance measure.

\section{Hybrid Improved Bacterial Swarm Optimization Algorithm}

The proposed Hybrid BFO-PSO algorithm was a combination of BFO and PSO algorithms. It was proposed to mitigate individual weaknesses in the BFO and PSO algorithms which were slow convergence and premature convergence, respectively (Shanmugasundaram Mohamed, \& Ruhaiyem, 2017b). There were three significant changes involved in the proposed hybrid BFO-PSO algorithm.

\section{Local best by Bacterial Foraging Optimization Algorithm}

In the proposed HBFO-PSO algorithm, the BFO algorithm was used to find the local best value. The BFO algorithm was affected by slow convergence. This was due to the fixed step size in the tumbling stage of the bacterium at the chemotaxis stage (Shanmugasundaram et al., 2017b). At the same time, however, it had the ability not to trap in local optima. Therefore, the BFO algorithm was used to find the local best value (pbest) whereas the global best search (gbest) was conducted by PSO. Further, the weakness of slow convergence was averted in BFO which is shown in Equation 1 and Equation 2 as follows.

$\theta i(j+1, k, l)=\theta i(j, k, l)+C(i) * \emptyset_{j}$

Pbest $=f(\Theta i(j+1, k, l))$

Where $\theta i(j+l, k, l)$ is the new position of the $i^{\text {th }}$ bacterium, $\theta i(j, k, l)$ previous position of the $i^{\text {th }}$ bacterium, $C(i)$-step size,$\varnothing_{j}$-previous direction of the $i^{\text {th }}$ bacterium and Pbest is the local best of fitness value of $\theta i(j+l, k, l)$ (Shanmugasundaram et al., 2017b).

\section{Adaptive Step Size in Tumbling Stage of Bacterium using Firefly Algorithm}

The bacterium in the BFO algorithm at the chemotaxis stage has two moves namely, swim and tumble. The swim is meant for moving the bacterium in the same direction whereas, the tumbling is meant for moving the bacterium in a random direction (Shanmugasundaram et al., 2017b). Step size C(i) is 
responsible for the tumble move of the $\mathrm{i}^{\text {th }}$ bacterium with a fixed step size within the range of between -1 and 1 . So, it delays in reaching the global solution. To accelerate the bacterium movement, in the proposed HIBS (Shanmugasundaram et al., 2017b), the fixed step size was changed into varying step sizes ranging from $[0,1]$ using the random walk procedure of the Firefly algorithm (Yang, 2009) to reach the optimum at the earliest convergence which is shown in Equation 3 and Equation 4.

$$
C(i)=\alpha(\text { rand }-1 / 2)
$$

Where $\alpha$ is the randomization variable, rand is a random number generator within the range from $[0,1]$. The step size $C(i)$ is deployed into the given below, which is responsible for the tumble move of the $i^{\text {th }}$ bacterium, (Shanmugasundaram et al., 2017b).

$$
\theta i(j+1, k, l)=\theta i(j, k, l)+C(i) * \emptyset j
$$

where $\theta i(j+l, k, l)$ is the new position of the $i^{\text {th }}$ bacterium, $\theta i(j, k, l)$-previous position of the $i^{\text {th }}$ bacterium, $C(i)$-step size, $\varnothing_{j}$-previous direction of the $i^{\text {th }}$ bacterium, (Shanmugasundaram et al., 2017b).

\section{Global Best by Particle Swarm Optimization Algorithm}

The PSO algorithm has an inherent disability of trapping local optima, but it has high convergence speed. Therefore, in the proposed hybrid algorithm, the PSO algorithm was deployed as mutation operator in the reproduction stage. It was used to find the global best search (gbest) by updating the position and directions of the $\mathrm{i}^{\text {th }}$ bacterium which is shown in Equation 5 and Equation 6.

$$
\begin{gathered}
\emptyset(j+1, k, l)=w * \emptyset(j)+c 1 * \text { rand } *(\text { pbest }-\Theta(i))+c 2 * \text { rand } *(\text { gbest }-\Theta(i)) \\
\Theta(j+1, k, l)=\Theta(j, k, l)+\emptyset(j+1, k, l)
\end{gathered}
$$

Where $\varnothing(j+1, k, l)$ - new direction of the ith bacterium, $\theta(j+1, k, l)$-new position of the $i^{\text {th }}$ bacterium, $w$-inertia weight, $c 1, c 2$ - acceleration constants, randrandom number between the range [0,1], pbest- local optimum value, gbestglobal optimum value, $\theta(j, k, l)$ previous position of the $i^{\text {th }}$ bacterium, $\varnothing(j)$ previous direction of the ith bacterium (Shanmugasundaram et al., 2017b). Positions and directions of the bacteria were updated by PSO algorithm only after the chemotaxis stage in which all the fitness evaluations were performed in the chemotaxis. The proposed algorithm is detailed in Figure 1. 
step 1. Begin

step 2. Initialize the $\mathrm{BFO}$ and $\mathrm{PSO}$ parameters

step 3. Do the Weighted sum score fusion

step 4. $\quad$ Sfi $=w^{*}$ Si $1+(1-w 1-w 3)^{*}$ Si2 $+(1-w l-w 2)^{*}$ Si3

step 5. Elimination-dispersal loop : For $1=0 ; 1<\mathrm{Ned} ; 1++$

step 6. Reproduction loop : For $\mathrm{k}=0$; $\mathrm{k}<\mathrm{Nre}$; $\mathrm{k++}$

step 7. Chemo taxis loop: For $\mathrm{j}=0 ; \mathrm{j}<\mathrm{Nc} ; \mathrm{j}++$

step 8.

For $\mathrm{i}=1$ to $\mathrm{N}$; $\mathrm{i}++$

step 9.

Compute Fitness : EER(i,j,k,l)

step 10.

Let EERlast $=\mathrm{EER}(\mathrm{i}, \mathrm{j}, \mathrm{k}, \mathrm{l})$

step 11.

Tumble: Step size $C(\mathrm{i})=\Theta(\mathrm{i}, \mathrm{j}, \mathrm{k})+\alpha($ rand-1/2)

step 12.

where rand $[0,1]$

step 13.

Move: $\Theta i(j+1, k, 1)=\Theta i(j, k, 1)+C(i)^{*} \varnothing j$

step 14 .

Swim: For $\mathrm{m}=0 ; \mathrm{m}<\mathrm{Ns}$; $\mathrm{m}++$

step 15.

if $\operatorname{EER}(\mathrm{I}, \mathrm{j}+1, \mathrm{k}, \mathrm{I})<$ EERlast

step 16.

Let EERlast $=\operatorname{EER}(1, \mathrm{j}+1, \mathrm{k}, \mathrm{l})$

step 17.

Compute $\Theta i(j+1, k, 1)=\Theta i(j, k, I)+C(i)^{*} Ø j$

step 18.

If $\Theta i(j+1, k, I)<$ pbest

step 19.

Let pbest $=\Theta i(j+1, k, I)$.

step 20.

Else

step 21.

Let $\mathrm{m}=\mathrm{Ns}$

step 22.

End of For statement

step 23.

End of For Statement for ith bacterium

step 24 .

End of chemotaxis loop

step 25.

Mutation by PSO operator

step 26.

$\varnothing(j+1)=w^{*} \varnothing(j)+c{ }^{*}$ rand ${ }^{*}($ pbest $-\Theta(i))+c 2^{*}$ rand ${ }^{*}($ gbest $-\Theta(i))$

step 27.

$\Theta i(J+1, k, 1)=\Theta i(j, k, 1)+\Theta(j+1, k, l)$

step 28. End of Reproduction loop

step 29. End of Elimination-dispersal loop.

step 30. If Iteration $<=\max (100)$, Go to Step 3

step 31. End

Figure 1. Proposed Hybrid Improved Bacterial Swarm (H IBS) Optimization Algorithm. 


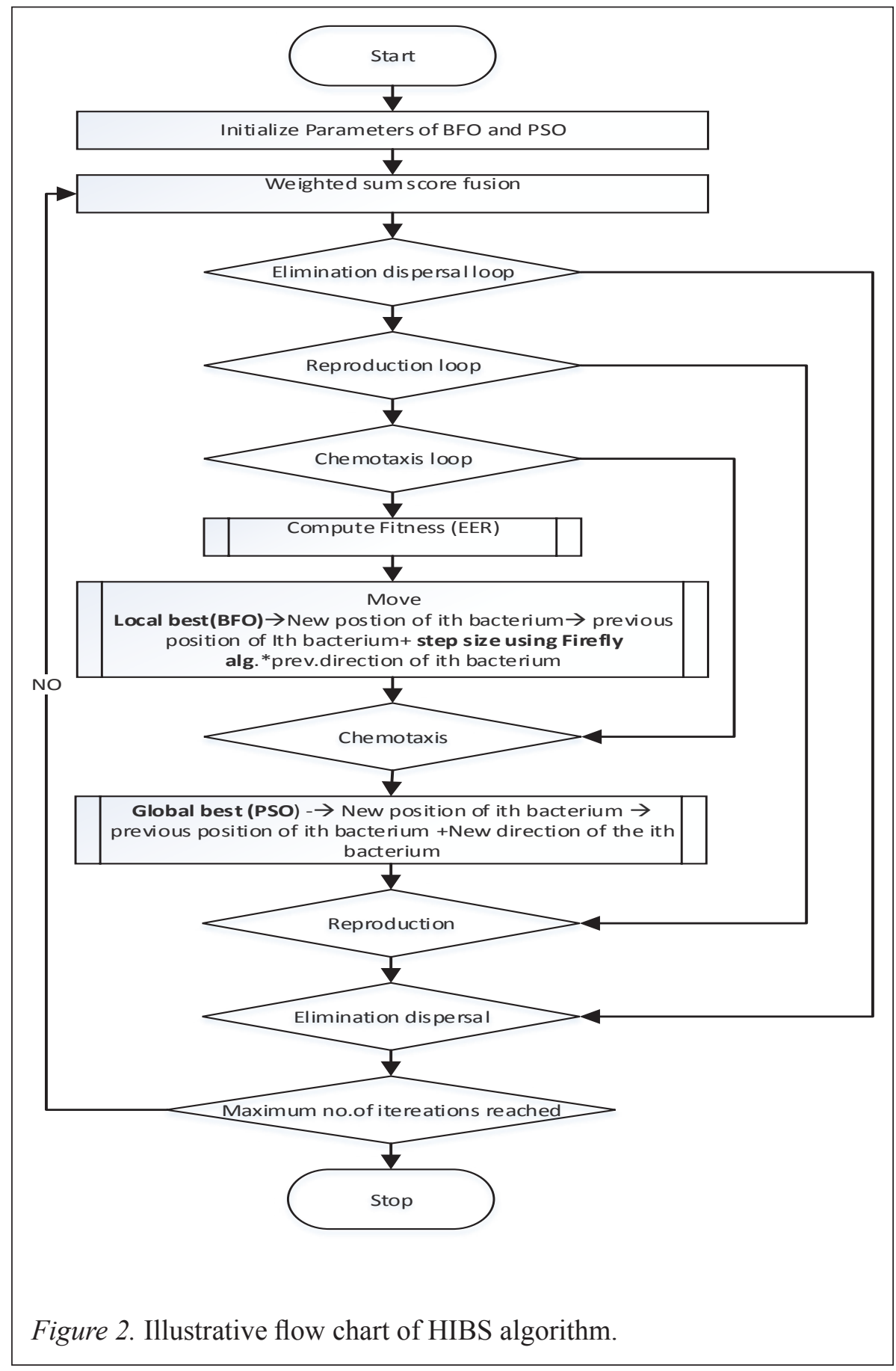


Figure 2 shows the illustrative flow chart of HIBS algorithm (Shanmugasundaram et al., 2017b). The hybridization of BFO and PSO forms the HIBS algorithm and also the fixed step size in the original BFO algorithm at the chemotaxis stage is changed to adaptive increasing step size (0.01) which range from 0 to 1 using Firefly algorithm.

\section{Hand-based Multibiometric Authentication System}

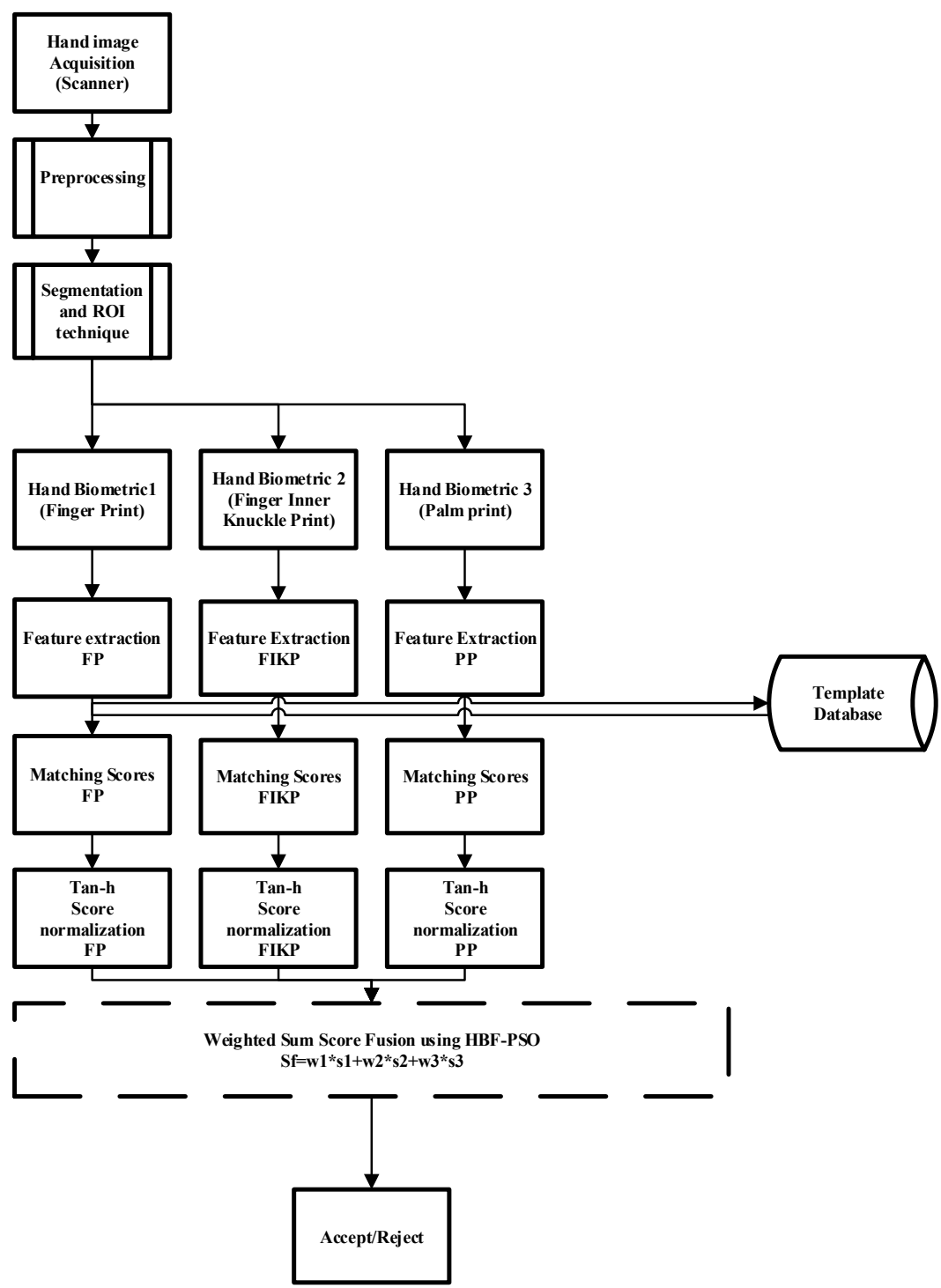

Figure 3. General framework of the hand-based biometric authentication system using HBF-PSO. 
The general framework design of the hand-based biometric authentication system is shown in Figure 3. First, the hand inner surface was scanned by a conventional scanner and after that image segmentation and Region of Interest (ROI) techniques (Bao, Zhang, \& Wu, 2005) were deployed to extract these three modalities: palm print, fingerprint, and finger inner knuckle print. After segmentation, preprocessing and feature extraction were conducted for all these modalities using spectral minutiae extraction (Kumar \& Wang, 2015). Then, the feature extracted images were stored in a template database for minutiae matching (Zafar, Ahmad, \& Hassan, 2014).

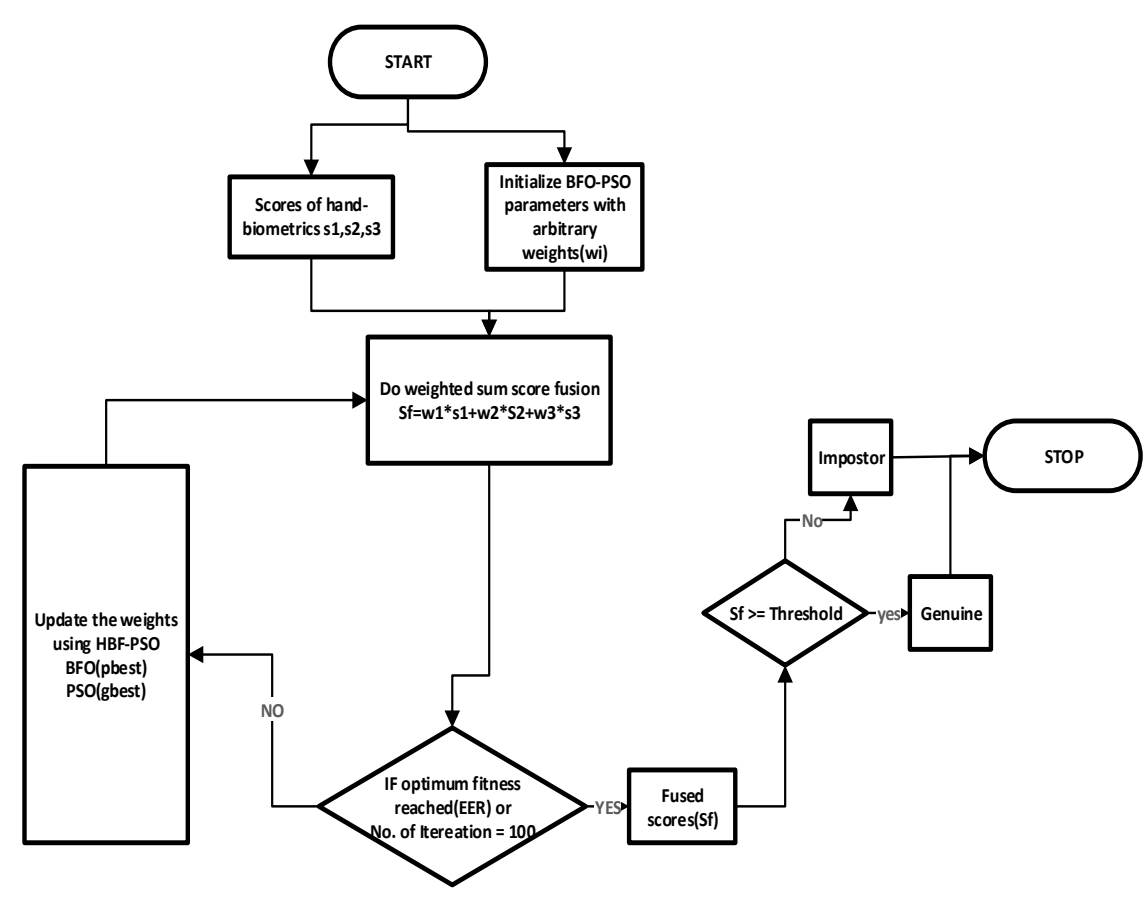

Figure 4. Weight optimization using proposed hybrid BF-PSO based weighted sum score fusion.

After that, the matching scores of all these modalities were generated by Euclidean distance matcher and deployed using tanh score normalization (Shanmugasundaram et al., 2017b) to check for similarity measures. If not, the corresponding scores would be transformed into a standard domain called tan-h score normalization by bringing the scores into the same range $[0,1]$. At the score level fusion, the weighted sum rule classifier was used for fusing 
the scores of different hand matchers after tan-h score normalization was completed. At this stage, the role of the hybrid meta-heuristic algorithm (HIBS) was to optimize weights associated with the fused modalities in the weighted sum score fusion to get the minimum EER value. Finally, the claimed identity was either accepted or rejected based on the fusion score value. If the fused score was higher than the threshold, then the user was accepted, otherwise it was rejected. This is shown in Figure 4.

\section{RESULTS AND DISCUSSION}

The Bosphorus hand image database (in Figure 5) was used for the experiment. There were 642 left and right human hand images with three poses for each hand. Therefore, a total of 3852 samples had been used for the multimodal biometric authentication system, out of which 828 samples of left hand images and 816 samples of right hand images were used for training (Shanmugasundaram et al., 2017b).

(a)

(b)
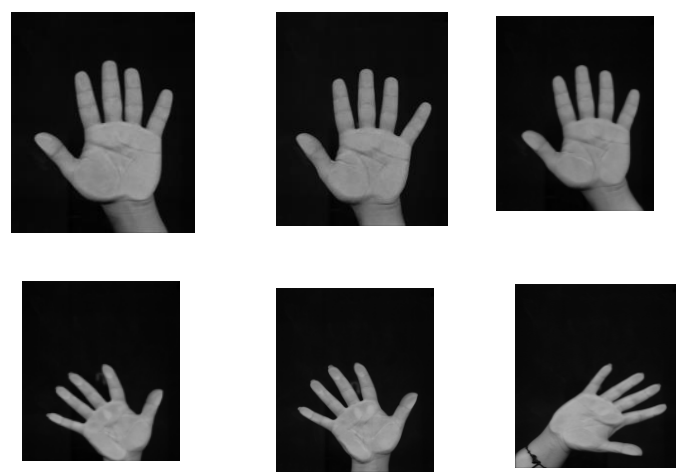

Figure 5. Sample images of (a) left hand and (b) right hand images of the same person (Shanmugasundaram et al., 2017b).

Figures 5 and 6 show the hand geometry segmentation of left and right hand images using the HBF-PSO algorithm and minutiae extraction which was also conducted for all three modalities namely, palm print, fingerprint and finger inner knuckle print, respectively. 


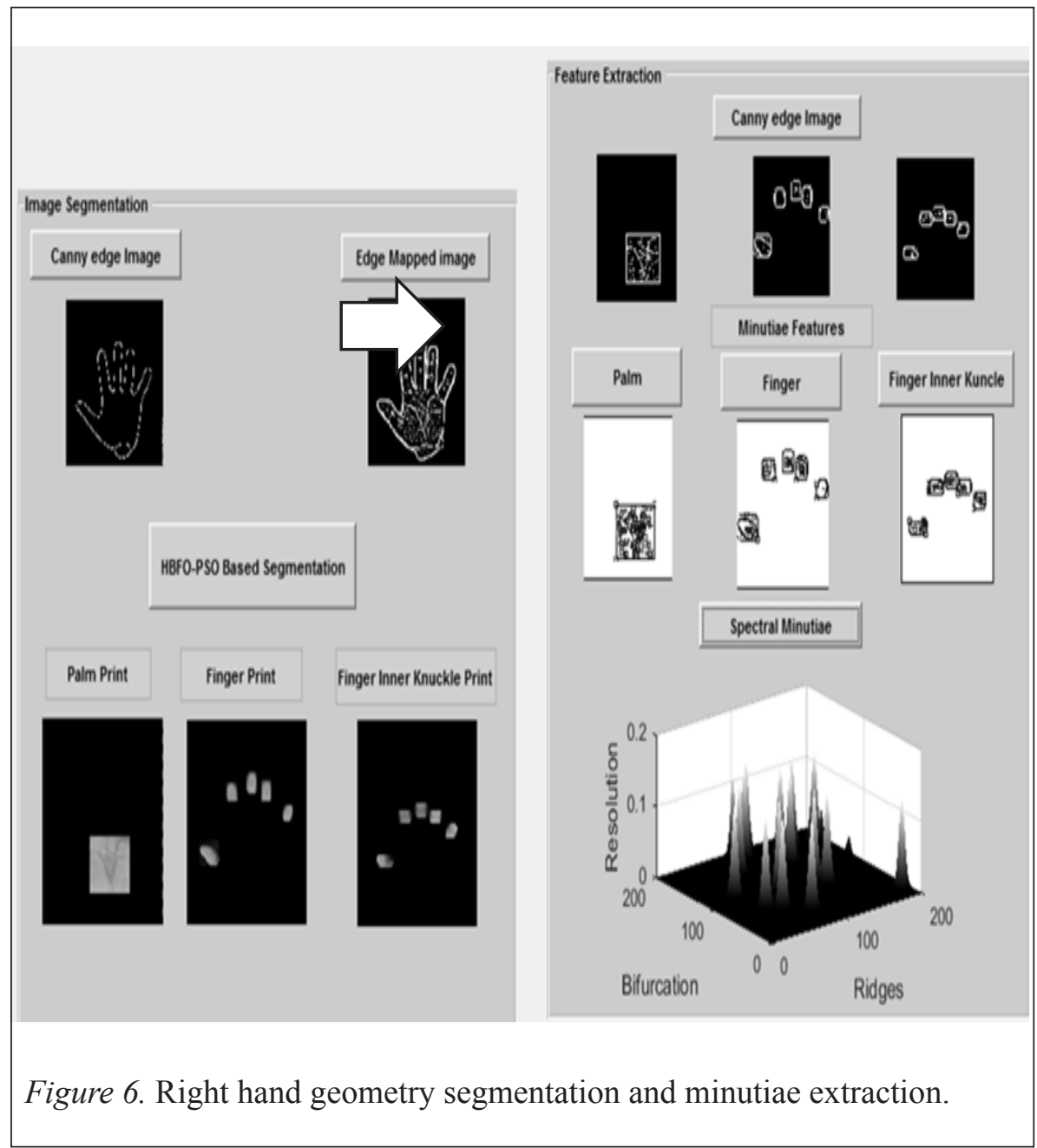

In order to test the effectiveness of the HIBS algorithm (Shanmugasundaram et al., 2017b), its performance was evaluated using four benchmark objective functions. Table 1 shows the performance of individual BFO and PSO algorithms in comparison with the HIBS algorithm. It can be observed that the hybrid BFO-PSO algorithm performed better than the original BFO and PSO algorithms on three benchmark functions except in the Ackley function. 


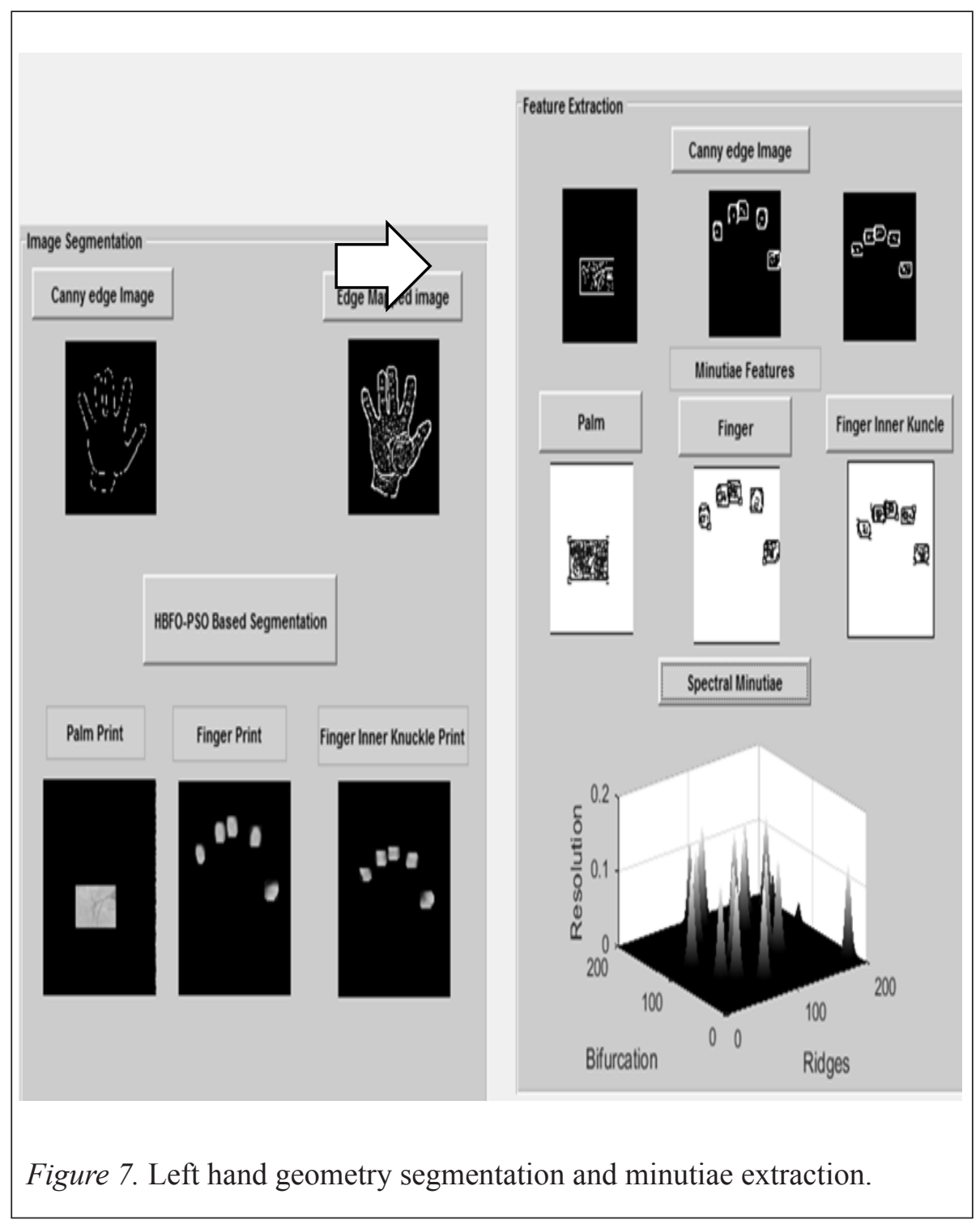

Similarly, Figure 7 shows the comparison of EER, FAR, and FRR values for the various hybrid algorithms. Weighted sum score fusion is used as fitness function. The HBF-PSO algorithm shows an exceptionally minimized EER value of 0.0036 as compared with other hybrid algorithms. 


\section{Table 1}

Results obtained for BFO, PSO, and HIBS(HBF-PSO) (Shanmugasundaram et al., 2017b) Algorithms using benchmark functions for the mean value of best cost.

\begin{tabular}{llll}
\hline Fitness Function & $B F O$ & $P S O$ & $H I B S$ \\
F1-Rosenbrock & 0.31628 & 0.63834 & $\mathbf{0 . 0 4 3 7 1}$ \\
F2- Ackley & 3.7583 & $\mathbf{2 . 3 8 4 5 2}$ & 3.2183 \\
F3- Griewank & 3.0254 & 3.8596 & $\mathbf{2 . 7 5 6 3}$ \\
F4-Rastrigin & 4.2356 & 6.29718 & $\mathbf{. 3 8 6 7 8}$ \\
\hline
\end{tabular}

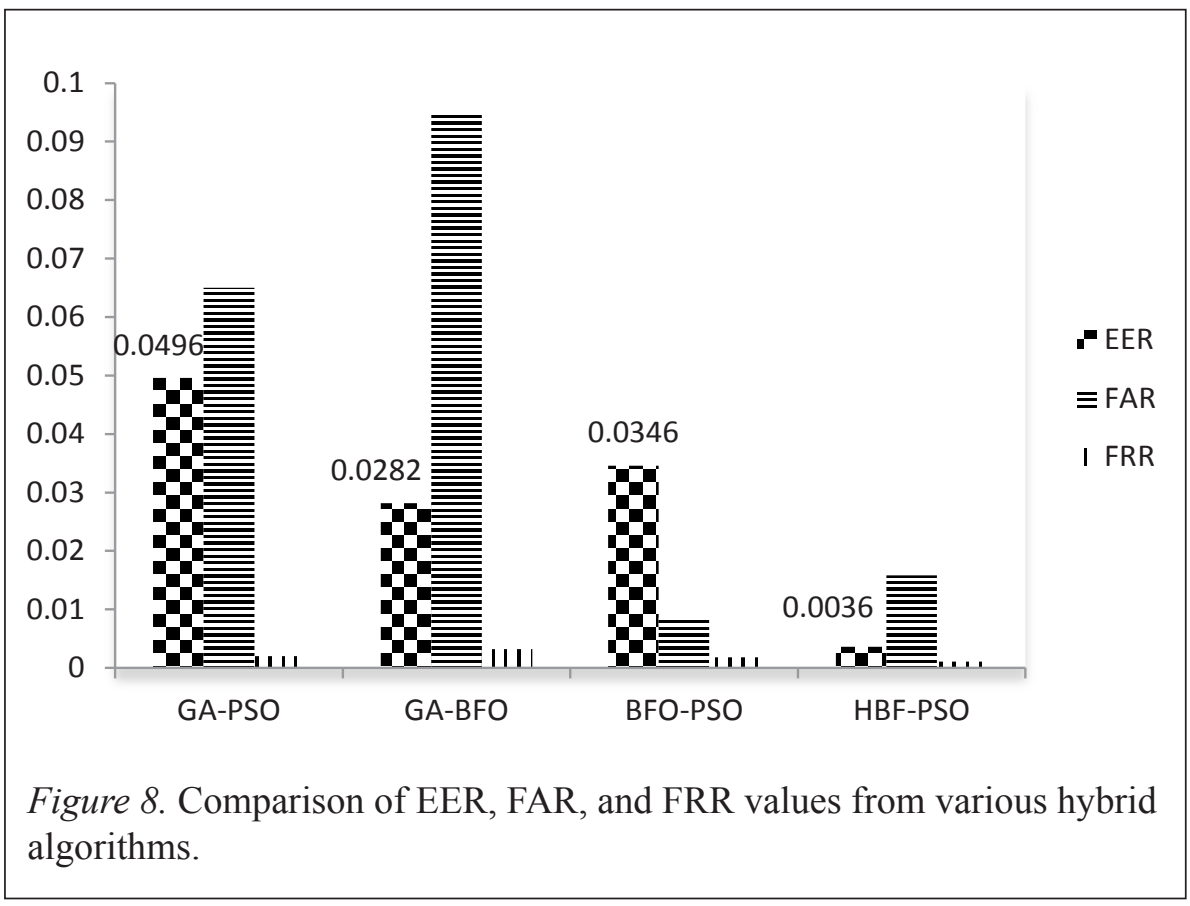

Table 2 presents the test results of GA-BFO, GA-PSO, BFO-PSO, and HIBS (Shanmugasundaram et al., 2017b) algorithms using benchmark functions as fitness functions and mean and standard deviation as parameters with the HIBS algorithm showing the best results. Similarly, in using the weighted sum score fusion as the fitness function, the HIBS algorithm reported the highest accuracy of $98.96 \%$ compared with other hybrid algorithms. The performance accuracy of the multimodal biometric authentication system was measured by plotting the ROC curves. The performance accuracy of the multibiometric 
system using the proposed HIBS algorithm was at $98.96 \%$. Here, the HIBS algorithm was used for optimizing weights and the weighted sum score fusion acted as the fitness function. This performance accuracy is plotted using the ROC curve against the GAR and FAR values, which is shown in Figure $8(\mathrm{a})$.

Figure 8(b) presents the error values against the threshold values by using the FAR vs. FRR graph. Here, the threshold values range from 0.0 and 1.0. The overlapping of FAR and FRR is considered as error rates regarding the threshold value. DET curve is another measure for analyzing error rates which is shown in Figure 8(c). The DET curve is plotted against the values of FAR and FRR.

Table 2

Results obtained for GA-PSO, GA-BFO, BFO-PSO, and HIBS (HBFPSO) (Shanmugasundaram et al., 2017b) Algorithms using benchmark functions and weighted sum score fusion as fitness functions.

\begin{tabular}{llrrrc}
\hline $\begin{array}{l}\text { Fitness } \\
\text { function }\end{array}$ & Parameter & GA-PSO & GA-BFO & BFO-PSO & HBF-PSO \\
Rosenbrock & Mean & 54.28 & 52.56 & 51.06 & 49.45 \\
& Std. deviation & 8.62 & 5.67 & 3.45 & 1.22 \\
Rastrigin & Mean & 24.28 & 22.56 & 19.06 & 18.08 \\
& Std. deviation & 5.62 & 5.67 & 4.89 & 3.45 \\
& Mean & 50.28 & 49.56 & 48.06 & 46.45 \\
Ackley & Std. deviation & 8.62 & 7.67 & 5.45 & 6.81 \\
& Mean & 24.28 & 22.56 & 17.06 & 15 \\
Griewank & Std. deviation & 8.62 & 5.67 & 3.45 & 1.99 \\
& Time (secs) & 178.32 & 161.86 & 257.13 & 223.23 \\
Weighted & Accuracy (\%) & 93.41 & 90.36 & 94.41 & 98.96 \\
$\begin{array}{l}\text { Sum Score } \\
\text { Fusion }\end{array}$ & & & & & \\
\hline
\end{tabular}




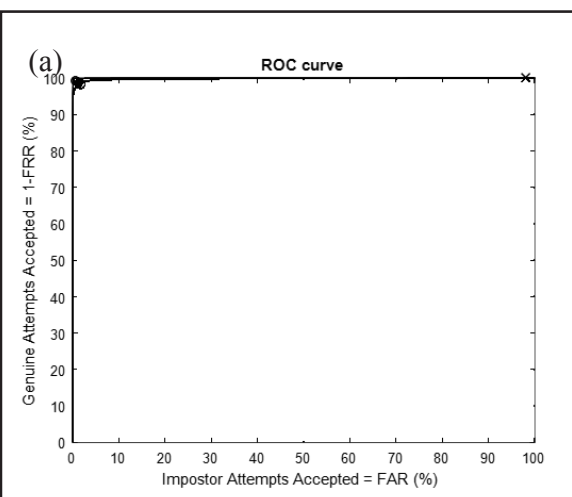

(b)
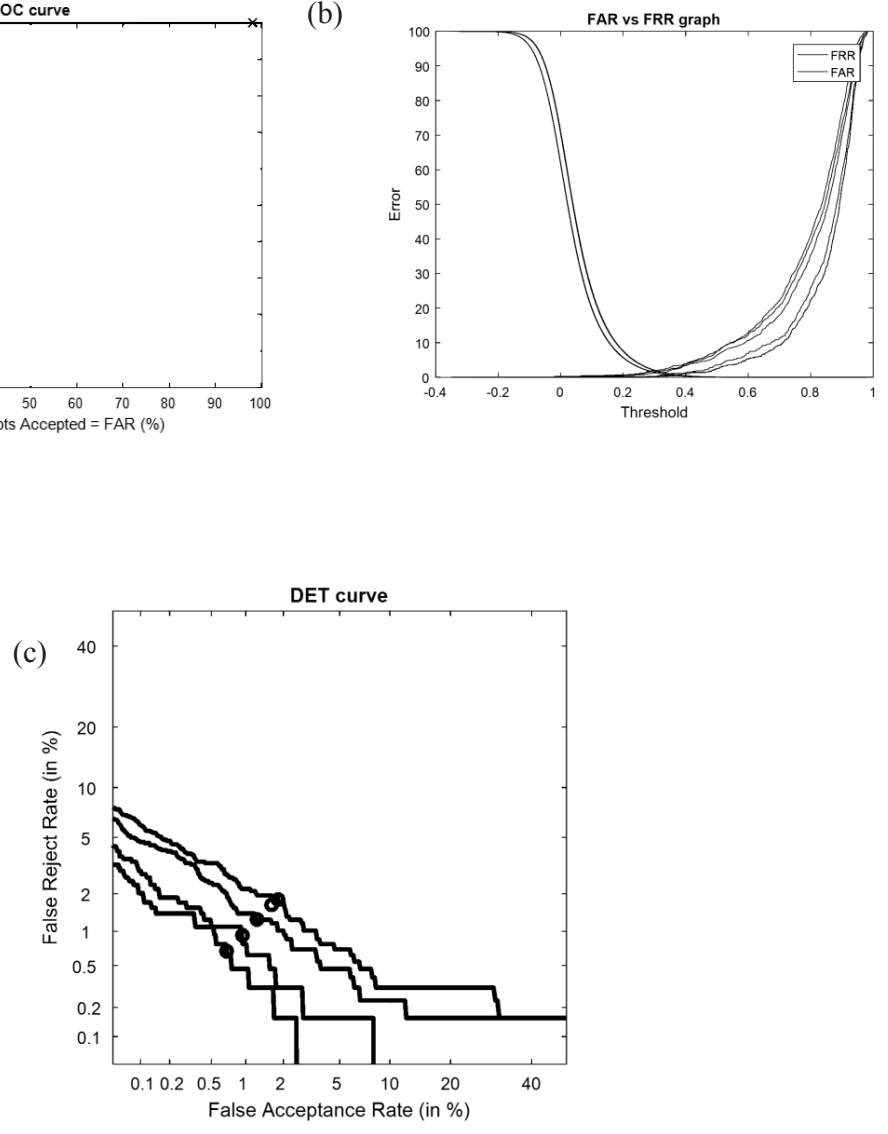

Figure 9. (a) ROC curve (b) FAR vs. FRR graph and (c) DET curve.

A comparison of performance measures of accuracy, EER, confidence intervals and threshold values of hand-based multibiometric authentication are shown in Table 3. The training and testing cases were performed with three folds of 272 images each by using single and double enrolments of right and left hand images from the Bosphorus database. Paired t-test was employed in the statistical hypothesis testing analysis for the HBF-PSO algorithm performance. Similarly, likelihood ratio hypothesis testing was conducted as the statistical analysis for score fusion to accept the user as a genuine person or to reject as an impostor for the hand-based multibiometric verification system. 
Table 3

Comparison of Performance Measures of Accuracy and EER Values with Confidence intervals and Threshold Values for Right and Left Hand Images from Bosphorus Database

\begin{tabular}{|c|c|c|c|c|c|c|}
\hline \multirow{9}{*}{ 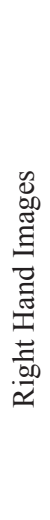 } & \multirow{5}{*}{ 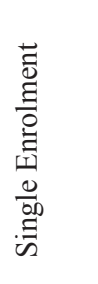 } & Parameter & First Fold & Second Fold & Third Fold & Average \\
\hline & & Accuracy & $96.96 \%$ & $96.49 \%$ & $95.71 \%$ & $96.39 \%$ \\
\hline & & EER & $1.64 \%$ & $1.25 \%$ & $1.81 \%$ & $1.56 \%$ \\
\hline & & $\mathrm{CI}$ & 0.30 & 0.29 & 0.31 & 0.26 \\
\hline & & Threshold & 0.2929 & 0.3092 & 0.2837 & 0.2953 \\
\hline & & Accuracy & $98.60 \%$ & $99.07 \%$ & $99.07 \%$ & $98.91 \%$ \\
\hline & $0 \quad \ddot{0}$ & EER & $0.0093 \%$ & $0.0065 \%$ & $0.0053 \%$ & $0.0070 \%$ \\
\hline & $\overrightarrow{\vec{z}} \frac{\Xi}{0}$ & $\mathrm{CI}$ & 0.32 & 0.27 & 0.27 & 0.29 \\
\hline & คี & Threshold & 0.3525 & 0.3729 & 0.3729 & 0.2953 \\
\hline & & Accuracy & $98.13 \%$ & $97.66 \%$ & $97.43 \%$ & $97.74 \%$ \\
\hline$\underset{\Xi}{\Xi}$ & & EER & $0.86 \%$ & $0.87 \%$ & $0.85 \%$ & $0.86 \%$ \\
\hline 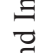 & $\stackrel{0}{00}$ & CI & 0.22 & 0.22 & 0.22 & 0.22 \\
\hline ज्ञ & 济䓅 & Threshold & 0.3007 & 0.2984 & 0.2985 & 0.2992 \\
\hline & & Accuracy & $99.22 \%$ & $99.68 \%$ & $99.89 \%$ & $99.59 \%$ \\
\hline & & EER & $0.0047 \%$ & $0.0036 \%$ & $0.0063 \%$ & $0.0049 \%$ \\
\hline & $\bar{\Xi}$ & $\mathrm{CI}$ & 0.23 & 0.19 & 0.19 & 0.20 \\
\hline & 式 & Threshold & 0.3599 & 0.3906 & 0.3906 & 0.3804 \\
\hline
\end{tabular}

\section{CONCLUSION}

In this paper, the HIBS algorithm was proposed to mitigate individual weaknesses of the classical BFO and PSO algorithms and to optimize weights associated with hand-based modalities in the weighted sum score fusion of hand-based multimodal biometric authentication system. The hand images were further deployed for segmentation into multimodalities like fingerprint, finger inner knuckle print, and palm print from the Bosphorous database. After segmentation and preprocessing, feature extraction was performed 
based on spectral minutiae recognition. Then score extraction was conducted based on Euclidean distance matcher. Tanh score normalization was deployed before score fusion. The extracted scores of these three modalities were then fused with optimal weights induced by the HIBS algorithm at the weighted sum score fusion of the hand-based multibiometric system. As a result, the equal error rates (EER) were drastically minimized to $0.0070 \%$ and $0.0049 \%$ from $1.56 \%$ and $0.86 \%$ for the right and left hand images of the Bosphorus database, respectively. The performance evaluations were conducted using ROC (Receiver Operating Characteristic) curve and DET (Detection Error Tradeoff) curve which were plotted against False Acceptance Rates (FAR) and False Rejection Rates (FRR), respectively. From the experimental results, it has been significantly proven that the proposed hybrid algorithm used in the hand-based multibiometric system showed a more exceptional performance in error minimization than other hybrid algorithms as discussed. Further, different databases related to hand-based images need to be tested in future work to enhance results.

\section{ACKNOWLEDGEMENT}

This work was funded by University Research Grant(1001/PKOMP/8014001), Universiti Sains Malaysia.

\section{REFERENCES}

Alford, A., Hansen, C., Dozier, G., Bryant, K., Kelly, J., Abegaz, T., \& Woodard, D. L. (2011). GEC-based multi-biometric fusion. IEEE Congress on Evolutionary Computation, (pp. 2071-2074). doi: 10.1109/ CEC.2011.5949870

Alostaz, A., \& Alhanjouri, M. (2013). A new adaptive BFO based on PSO for learning neural network. i-Manager's Journal on Computer Science, 1(3), 9. doi: 10.26634/jcom.1.3.2544

Bakwad, K. M., Pattnaik, S. S., Sohi, B. S., Devi, S., Panigrahi, B. K., Das, S., \& Lohokare, M. R. (2009). Hybrid bacterial foraging with parameter free PSO. World Congress on Nature \& Biologically Inspired Computing, (pp. 1077-1081). doi: 10.1109/NABIC.2009.5393867

Bao, P., Zhang, L., \& Wu, X. (2005). Canny edge detection enhancement by scale multiplication. IEEE Transactions on Pattern Analysis and Machine Intelligence, 27(9), pp. 1485-1490. 
Biswas. A., D. S., Das, S., \& Abraham, A. (2007). Synergy of PSO and bacterial foraging optimization: BA comparative study on numerical benchmarks. Advances in Soft Computing. 44:255-263. doi: 10.1007/978-3-54074972-1_34

Cherifi Dalila, Hafnaoui Imane \& Nait-Ali Amine (2015). Multimodal score-level fusion using hybrid GA-PSO for multibiometric system. Informatica, 39(2), 837-913.

Daas, M. S., Chikhi, S., \& Batouche, M. (2015). Bacterial foraging optimization with double role of reproduction and step adaptation. Proceedings of the International Conference on Intelligent Information Processing, Security and Advanced Communication, 71. doi: $10.1145 / 2816839.2816868$

Eberhart, R., \& Kennedy, J. (1995). A new optimizer using particle swarm theory. Proceedings of the Sixth International Symposium on Micro Machine and Human Science, 39-43. doi: 10.1109/MHS.1995.494215

Gabi, D., Ismail, A. S., Zainal, A. Zakaria, Z., \& Al-Khasawneh, A. (2018). Hybrid cat swarm optimization and simulated annealing for dynamic task scheduling cloud computing environment. Journal of ICT, 17(3), 435-467.

Hanmandlu, M., Kumar, A., Madasu, V. K., \& Yarlagadda, P. (2008). Fusion of hand based biometrics using particle swarm optimization. Proceedings of the Fifth International Conference on Information Technology: New Generations, 783-788. doi: 10.1109/ITNG.2008.252

Jain, A. K., Nandakumar, K., \& Ross, A. (2016). 50 years of biometric research: Accomplishments, challenges, and opportunities. Pattern Recognition Letters, 79, 80-105. doi: 10.1016/j.patrec.2015.12.013

Jarraya, Y., Bouaziz, S., Alimi, A. M., \& Abraham, A. (2013). A hybrid computational chemotaxis in bacterial foraging optimization algorithm for global numerical optimization. Proceedings of the IEEE International Conference on Cybernetics (CYBCO), pp. 213-218. doi: 10.1109/CYBConf.2013.6617428

Kennedy, J., Kennedy, J. F., Eberhart, R. C., \& Shi, Y. (2001). Swarm intelligence: The Morgan Kaufmann Series. ISBN: 978-1558605954

Kora, P., \& Kalva, S. R. (2015). Hybrid bacterial foraging and particle swarm optimization for detecting Bundle Branch Block. SpringerPlus, 4(1), 1. doi: 10.1186/s40064-015-1240-Z

Kumar, A., \& Wang, B. (2015). Recovering and matching minutiae patterns from finger knuckle images. Pattern Recognition Letters, 68(2), 361367. doi: 10.1016/j-patrec.2015.08.013

Passino, K. M. (2002). Biomimicry of bacterial foraging for distributed 
optimization and control. IEEE Control Systems, 22(3), 52-67. doi: 10.1109/MCS.2002.1004010

Ross, A. A., Nandakumar, K., \& Jain, A. (2006). Handbook of multibiometrics (Vol. 6): Springer Science \& Business Media.

Shanmugasundaram, K., Mohamed, A. S. A., \& Ruhaiyem, N. I. R. (2017a). Hybrid improved bacterial swarm (HIBS) optimization algorithm. Proceedings of the International Conference of Visual Informatics. Springer, 71-78. doi: 10.1007/978-3-319-70010-6_7

Shanmugasundaram, K., Mohamed, A. S. A., \& Ruhaiyem, N. I. R. (2017b). An overview of hand-based multimodal biometrie system using multiclassifier score fusion with score normalization. Proceedings of the International Conference on Signal Processing and Communication, 53-57. doi: 10.1109/CSPC.2017.8305806

Shehab, M. Khader, A. T., \& Laouchedi, M. (2018). A hybrid method based on cuckoo search algorithm for global optimization problems. Journal of ICT, 17(3), 469-491.

Yan, X., Zhu, Y., Chen, H., \& Zhang, H. (2012). Improved bacterial foraging optimization with social cooperation and adaptive step size. Proceedings of the International Conference on Intelligent Computing, 634-640. doi: 10.1007/978-3-642-31588-6_81

Yang, X. S. (2009). Firefly algorithms for multimodal optimization. Proceedings of the International Symposium on Stochastic Algorithms, 169-178. doi: 10.1007/978-3-642-04944-6_14

Zafar, W., Ahmad, T., \& Hassan, M. (2014). Minutiae based fingerprint matching techniques. Proceedings of the IEEE Seventeenth International Conference on Multi-Topic, 411-416. doi: 10.1109/ INMIC.2014.7097375 